\section{$\underset{\substack{\text { hommes } \\ \text { \& migrations }}}{ }$}

\section{Hommes \& migrations}

Revue française de référence sur les dynamiques

migratoires

$1301 \mid 2013$

Migrations et mondes ruraux

\title{
Naissance du kaneka
}

\section{François Bensignor}

\section{(2) OpenEdition \\ Journals}

\section{Édition électronique}

URL : http://journals.openedition.org/hommesmigrations/1959

DOI : 10.4000/hommesmigrations.1959

ISSN : 2262-3353

\section{Éditeur}

Musée national de l'histoire de l'immigration

\section{Édition imprimée}

Date de publication : 1 janvier 2013

Pagination : 198-204

ISBN : 978-2-919040-21-6

ISSN : $1142-852 X$

\section{Référence électronique}

François Bensignor, « Naissance du kaneka », Hommes \& migrations [En ligne], 1301 | 2013, mis en ligne le 29 mai 2013, consulté le 22 septembre 2020. URL : http://journals.openedition.org/ hommesmigrations/1959; DOI : https://doi.org/10.4000/hommesmigrations.1959

Ce document a été généré automatiquement le 22 septembre 2020.

Tous droits réservés 


\title{
Naissance du kaneka
}

\author{
François Bensignor
}

1 La Nouvelle-Calédonie est devenue un territoire d'outre-mer en 1946, après un siècle d'une colonisation très dure pour les Kanak, ses premiers habitants. S'ils y acquièrent la citoyenneté française, puis le droit de vote en 1957, leur discrimination perdure jusqu'à ce qu'ils décident de faire cession. En prenant la tête du "gouvernement provisoire de Kanaky" le $1^{\text {er }}$ décembre 1984, Jean-Marie Tjibaou est bien conscient qu'il engage son peuple dans une véritable révolution culturelle. La musique va y jouer un rôle de premier plan vis-à-vis des jeunes générations, qui s'exprimeront et se reconnaîtront à travers ce nouveau genre appelé "kaneka".

2 Dans les années 1950-1960, la reine des dancings de Nouméa est la musique tahitienne, avec ses valses et la douceur de ses harmonies. La société calédonienne dans toute sa diversité l'a adoptée. La plupart des musiciens qui se produisent sur scène sont tahitiens, fidjiens, maoris, etc. Leur maîtrise de l'art et de la technique musicale est un modèle pour les Calédoniens qui veulent se lancer dans les musiques modernes. Dans cette capitale à la population cosmopolite, les cultures se croisent et se côtoient, parfois aussi sur le mode de la confrontation.

\section{Montravel}

3 Les jeunes de la communauté mélanésienne de Nouméa s'identifient au pop-rock des Beatles ou des Rolling Stones, à la soul music des James Brown et Otis Redding. En 1968, alors qu'une pléiade d'artistes honorent à New York la mémoire de Martin Luther King, émerge le premier groupe de jeunes Kanak urbains: Wax. Adeptes de la soul, ses fondateurs, les frères Xenie, ouvrent la voie de la musique électrique aux jeunes du quartier de Montravel.

D'abord champ de tir, puis réserve pénitentiaire, l'espace militaire de Montravel laisse place dans les années 1960 à la cité mélanésienne, quartier d'habitat social destiné à la population kanak venue de l'intérieur de la Grande Terre et des îles en quête de travail. La cité Pierre-Lenquête, achevée en 1970, comprend une vingtaine d'immeubles et jouxte celle où la Société Le Nickel (SLN) loge ses ouvriers. "La cité de Montravel a été 
construite en face du dépotoir, exposée aux poussières de l'usine de nickel toute proche", explique Warawi Wayenece, dont la famille, originaire de l'île de Maré, apprécie toutefois d'accéder à un logement neuf.

5 Déracinés, loin de leurs tribus, les habitants de Montravel sont dans une situation de double entre-deux. D'un côté, ils essayent de préserver les traditions spécifiques à leur région, à leur tribu, de l'autre, ils sont entourés de communautés venues des aires culturelles de toute la Nouvelle-Calédonie, avec leurs langues, leurs coutumes particulières. Enfin, ils sont confrontés au mode de vie occidental qui s'impose à Nouméa, ainsi qu'aux préjugés raciaux. "À la différence des Kanak qui vivaient dans les tribus, nous étions en butte à l'exclusion, poursuit Warawi Wayenece. Cette pression psychologique faisait aussi que le jeune Kanak élevé en milieu urbain était beaucoup plus éveillé à l'injustice. Dès que l'on passait la porte de notre maison, on se retrouvait confronté à l'exclusion sociale. Mais cette expérience de la souffrance s'est avérée fertile sur le plan de la créativité."

6 En baptisant leur nouveau groupe Black People, les frères Xenie sont déjà dans la revendication de leur différence et d'une reconnaissance de leur identité de "gens de couleur". Le message des Black Panthers, tout comme l'affirmation sans complexe du “ black and proud" (noir et fier de l'être) décliné dans la chanson du "Soul Brother" James Brown et dans les films du genre "blackspoitation", sont intégrés par cette jeunesse urbaine, comme l'explique le musicien et compositeur Jimmy Oedin, ex-guitariste des Black People: "La lutte des Afro-Américains a servi de détonateur au mouvement de prise de conscience et de revendication identitaire des Kanak. La soul et le funk sont des sources d'inspiration extrêmement prisées en Nouvelle-Calédonie."

7 Le jeune public kanak, notamment dans les bals, apprécie également le rock, comme l'atteste le succès de Yata, autre groupe issu de Montravel. Fondé en 1978 par Théo Ménango, Yata traduit parfaitement l'esprit revendicatif qui va s'amplifiant à la fin des années 1970. Contrairement à la plupart des autres groupes mélanésiens, qui affichent une homogénéité sinon familiale, du moins tribale ou linguistique, Yata est composé de musiciens issus de différentes communautés résidant à la cité. Tous les jeunes qui ont envie de bouger se reconnaissent dans sa musique, qu'ils soient mélanésiens, javanais, wallisiens, caldoches ou européens.

\section{Le patrimoine}

8 Après avoir marqué les esprits par son action culturelle, notamment grâce au succès de Mélanésia $2000^{1}$, Jean-Marie Tjibaou fait une entrée brillante en politique en 1977, devenant maire de Hienghène, commune la plus proche de sa tribu de Tiendanite, puis vice-président de l'Union calédonienne (UC) et conseiller territorial. À la tête du gouvernement régional entre 1982 et 1985, il peut développer sa vision politique et culturelle, mettant en place notamment deux structures qui valorisent la conception du monde kanak : l'Office culturel scientifique et technique kanak (OCSTK), qui deviendra l'Agence de développement de la culture kanak (ADCK) après les accords de Matignon, et l'office foncier, qui deviendra l'Agence de développement rural et d'aménagement foncier (Adraf). “Autour du gouvernement Tjibaou s'articule l'organisation du Festival des arts du Pacifique, consacré au patrimoine des sociétés océaniennes, explique Emmanuel Tjibaou, fils du leader politique et actuel directeur du centre culturel qui porte son nom. Ce festival doit être organisé en 1984 à Nouméa, et une campagne de recherche est engagée dès 1982 
par l'OCSTK sur le patrimoine traditionnel : chant, danse, musique, rituels, archéologie, discours, techniques, etc."

Parmi les collecteurs qui participent à l'inventaire patrimonial des chants et des danses figure Gilbert Tein. Jeune musicien originaire de la chaîne de montagnes qui s'élève derrière Hienghène, il a fait ses premières chansons engagées pour l'élection de JeanMarie Tjibaou, qui l'encourage à persévérer dans ses créations. À cette époque, lorsque Gilbert pense musique, il ne lui vient pas à l'esprit d'y inclure les chants traditionnels que perpétuent quelques vieux. Dans sa langue nemi - comme dans les autres langues kanak -, il n'existe pas de mot pour exprimer le fait de faire de la musique, alors qu'il en existe pour "danser" et "chanter".

Petit-fils de pasteur, Gilbert Tein a chanté le "do" en chorale à l'église et il estime qu'il ne peut pas chanter les chants aé aé, réservés aux cérémonies traditionnelles. L'ethnomusicologue Jean-Michel Beaudet, venu travailler sur les traditions musicales des tribus kanak, va lui ouvrir les yeux avec cette question simple : "Comment se fait-il que vous, les jeunes Kanak, ne chantiez plus comme le font vos anciens, dont vous collectez les chants?" Un univers de réflexion s'ouvre alors pour le jeune Gilbert, qui ne l'a plus refermé depuis.

11 En même temps qu'il prend conscience de la valeur de ce que véhiculaient les voix des anciens, il comprend que les espaces appropriés à l'expression de ces formes culturelles ont disparu. "Il n'y a jamais eu de structure traditionnelle posant comme un devoir ou une contrainte l'apprentissage du chant aé aé. Mes grands-pères et grands-oncles chantaient un répertoire traditionnel très étendu. Mais ils chantaient également à l'église", explique Gilbert Tein. Ainsi, progressivement, sa démarche de musicien-créateur va-t-elle se structurer autour de l'idée de renverser cette absence d'intérêt de la jeunesse pour les chants traditionnels qui ne leur ont pas été transmis. Le processus se développera sur le temps long et en profondeur.

\section{L'hymne}

12 Pour l'équipe culturelle du Conseil de gouvernement territorial, constituée de Jacques Iekawé, Jean-Marie Tjibaou et Yeiwéné Yeiwéné, l'accueil du Festival des arts du Pacifique à Nouméa en 1984 est un enjeu majeur. Il permet en effet de resserrer les liens avec les vingt-cinq nations de la zone Pacifique, qui ont pris fait et cause contre l'obstination des gouvernants français à poursuivre les essais nucléaires sur l'atoll de Mururoa. Il est donc primordial de donner une couleur très "Pacifique" à la représentation calédonienne.

Un concours est organisé dans les pays participants pour créer l'hymne du festival. Jacques Iekawé jette son dévolu sur Warawi Wayenece, qui vient d'être embauché à l'OCSTK. L'homme politique connaît le jeune musicien pour lui avoir fait bénéficier d'une bourse d'étude au conservatoire de Metz. De retour à Nouméa, Warawi avait formé avec ses frères le groupe Amakal, dont la folk mélanésienne avait reçu les faveurs du public. Reparti en France à la fin des années 1970, on lui proposa d'enseigner quelque temps sa langue, le nengone, à l'Institut national des langues et civilisations orientales (Inalco) à Paris. Dans ce cadre, il bénéficie des cours du professeur Tran Van Khê, éminent musicologue vietnamien, qui oriente le travail du jeune Kanak sur la transposition écrite des harmonies non tempérées chantées par les chorales 
traditionnelles de Maré. En regagnant son archipel, Warawi Wayenece met en pratique ses recherches musicales.

La pièce qui en résulte va servir de base à la musique de l'hymne. Pour l'enregistrement, le musicien fait appel à ses amis de Montravel, Théo Menango et quelques-uns des membres de Yata. Mais comment faire admettre à ces jeunes gens fascinés par la fougue de Jimi Hendrix qu'ils doivent utiliser des instruments que leurs anciens préparent avec des matériaux cueillis dans la nature et qui servent seulement le temps des cérémonies et danses rituelles? Pour ces enfants du béton, bambous ou paquets de feuilles appartiennent à un monde révolu dont ils entendent s'échapper pour de bon. Cependant, les commanditaires politiques ont bien précisé qu'ils veulent une musique contemporaine représentant l'identité de la culture kanak. Et comment l'exprimer si ce n'est avec des éléments qui en sont les principaux marqueurs? Les rockers de Montravel n'ont d'autre choix que de s'exécuter.

Ils vont même trouver une satisfaction particulière à présenter leur création sur la scène du Liberty, salle de cinéma devenue mythique à Nouméa pour avoir accueilli des concerts de légende. Car cette fois, le groupe fait face à d'autres réticences, comme le raconte Wayari Wayenece : "Lorsqu'on a demandé aux gens venus des tribus de chanter avec des instruments traditionnels, ils ont eu peur. Ils disaient que le pasteur ne serait pas d'accord. Ils voulaient chanter des tempérances!..." La chape de plomb de l'évangélisation a banni toute référence aux expressions rituelles propres à la société mélanésienne : une sorte de malédiction menace toute tentative de renouer avec cette "sauvagerie" des temps anciens.

16 "C'est donc un jeune de la cité mélanésienne, arborant crânement un masque kanak, qui empoigne les percussions d'écorce, les entrechoque avec ferveur, exposant à l'avant-scène sa fière identité, poursuit Wayenece. Cette fougue, seuls les Kanak élevés en milieu urbain étaient capables de l'exprimer. Le kaneka n'aurait pas pu naître dans les tribus. Parce qu'il résulte d'une souffrance consécutive à l'exclusion, à la ségrégation, à la discrimination des Kanak dans la promiscuité de la ville. En revanche, le kaneka allait permettre aux jeunes vivant en brousse de se projeter dans l'avenir."

\section{Kanaky}

17 L'hymne sélectionné pour le Festival des arts du Pacifique préfigure par sa conception la musique que l'on nommera bientôt le "kaneka". Mais il ne sera joué devant les délégations venues d'Océanie qu'en 1985 à Tahiti. En effet, la situation insurrectionnelle qui s'empare de la Nouvelle-Calédonie avec le boycott actif des élections territoriales du 18 novembre 1984 oblige à reporter l'événement. On entre alors dans ce que les médias français appellent pudiquement les "événements", qui sont en fait une quasi-guerre civile sur fond d'exclusion culturelle et raciale. Prolongeant les effets désastreux de la colonisation, communauté européenne et forces de l'ordre affrontent les Kanak excédés de se voir toujours niés dans leur identité et leur culture.

Les passions se cristallisent autour du nouveau statut élaboré pour la NouvelleCalédonie par le secrétaire d'État à l'outre-mer, Georges Lemoine. Il offre une grande autonomie au territoire, le préparant à un référendum d'autodétermination que réclament les indépendantistes. Mais ces derniers ne peuvent accepter que tous les habitants du territoire aient le droit de voter: le grand nombre de nouveaux immigrants européens et océaniens arrivés récemment minorise en effet la 
participation des Kanak. Quant aux anti-indépendantistes, ils estiment que ce statut, qui accroît largement les pouvoirs du gouvernement territorial, induit le risque d'une séparation entre la France et la Nouvelle-Calédonie: une éventualité pour eux inconcevable.

Mis en place le 6 septembre 1984, le "statut Lemoine" est rejeté par les deux camps. Deux semaines plus tard, Jean-Marie Tjibaou transforme le FI en Front de libération nationale kanak et socialiste (FLNKS), qui agrège deux nouveaux adhérents représentant les syndicats et les femmes militantes. Les élections territoriales, boycottées par plus de la moitié des électeurs, mais remportées par le Rassemblement pour la Calédonie dans la République (RPCR), entraînent les premières violences.

Le $1^{\text {er }}$ décembre 1984, Jean-Marie Tjibaou prend la tête du "gouvernement provisoire de Kanaky" formé avec ses amis politiques. En hissant pour la première fois les couleurs de la Kanaky - le drapeau bleu, rouge, vert, orné d'un cercle jaune où se dresse une flèche faîtière -, le leader du FLNKS entonne son poème "Kanaky mon pays", qui sera mis en musique par Gilbert Tein et son groupe Bwanjep. Quatre jours plus tard, deux des frères Tjibaou et huit de leurs compagnons, au retour d'une réunion politique, sont tués au cours d'une embuscade sur la route qui mène à Tiendanite. Cet événement exacerbe les tensions, d'autant que les coupables, libérés moins d'un an après les faits, sont acquittés par la cour d'assises de Nouméa l'année suivante. Coupée en deux entre 1984 et 1988, la Nouvelle-Calédonie vit une période de heurts et de violences d'État, pendant qu'un formidable bouillonnement culturel saisit l'ensemble du monde kanak, dont il sort transformé à jamais.

\section{L'outil de la culture}

21 Au début des années 1980, la diffusion de musique kanak est cantonnée aux événements traditionnels: coutumes, mariages, deuils, naissances, bonne année... Aucun autre espace public de diffusion n'existe, jusqu'à la création du centre culturel de Hienghène, fin 1984. "Au centre culturel de Hienghène, j'ai vu pour la première fois des groupes jouer sur des guitares sèches et des percussions traditionnelles en écorce de figuier, se souvient Emmanuel Tjibaou. J'ai $v u$ aussi des ensembles traditionnels. Toutes les manifestations politiques étaient accompagnées par des chants traditionnels: la coutume pour accueillir les gens l'imposait. Pour que l'échange soit nourri de la réflexion, il faut intercéder auprès des esprits afin qu'ils favorisent l'échange et donnent leur bénédiction."

Le deuxième fils de Jean-Marie est né en 1976. Sa jeunesse a été bercée par les chants aé aé de la région nord-est de la Grande Terre. "J'étais curieux, dit-il. Les vieux que l'on faisait venir étaient de ma famille, originaires de Ponérihouen pour celle de ma mère, Marie-Claude, ou de Hienghène pour celle de mon père. Pendant que mes parents discutaient de politique, j'allais avec les vieux qui blaguaient et qui nous expliquaient. Au moment du 'coup de feu', il fallait courir pour faire les instruments traditionnels, tenir les micros. Et pendant les manifestations, aller chercher les pierres de fronde..."

L'éducation qu'il reçoit l'amène à appréhender et à comprendre cette culture traditionnelle que son père s'efforce de valoriser par tous les moyens, parce qu'il en va de la survie de son peuple. "Il faut se rappeler d'où l'on vient, explique Emmanuel. À Nouméa, tout ce qui était rattaché à la culture kanak était considéré comme relevant du domaine de la sauvagerie, os dans le nez et anthropophagie. Pour les colons, les Kanak n'avaient pas de civilisation, ne chantaient pas mais émettaient des sons, n'avaient pas de vocabulaire 
mais communiquaient par gestes. Ces préjugés ont encore cours chez une partie de la population de Nouvelle-Calédonie. Le combat pour la musique s'est articulé autour de ce genre d'affirmation. Le fait de bousculer les vieux pour leur demander de venir chanter en dehors des cérémonies coutumières dans le cadre de manifestations ou festivals était très nouveau pour nous. Nous étions obligés de demander des danses et des chants en expliquant clairement les finalités qui sont celles de l'édification d'une société qui nous reconnaisse pour nos valeurs." La mise en place du centre culturel de Hienghène a pour but non seulement de mobiliser autour d'une politique et d'une culture, mais aussi de faire évoluer les modes de relations entre communautés dans l'espace traditionnel mélanésien. "Si les Kanak veulent se définir comme faisant partie d'un pays indépendant, ils doivent se considérer comme un groupe homogène, poursuit Emmanuel Tjibaou. Pour cela, nous sommes obligés de nous détacher des relations qui ont prévalu pendant près de deux mille ans: la chefferie, le découpage par clans de la société, le mariage exogame, le tout étant formalisé par des échanges cérémoniels, des chants, des danses, des rituels. Les mouvements politiques se sont toujours fondés sur la coutume et sur les relations entre les chefferies, la nécessité de se reconnaître d'abord entre Kanak. Les relations d'alliances étaient nécessaires à l'unification dans la revendication politique et culturelle."

\section{Le nom}

La création du terme "kaneka", c'est un peu une histoire de poule et d'œuf... Évidemment, le mot vient de "Kanak", transformation du mot "Canaque", autrefois une insulte. C'est une référence identitaire du peuple qui va donner à son pays le nom de Kanaky. Mais, au pays où la coutume offre un espace indispensable de négociation, d'échange et de partage formel, les mots sont lourds de sens. Ils ne peuvent pas être bradés, ni sifflés dans le vent. Il est donc important que la problématique soit posée, analysée, discutée, et que chacun y apporte sa pierre.

C'est ce qui est fait à Canala en 1986, lors des Rencontres tradition et création, qui réunissent des membres des groupes Bwafo, Bwanjep, Yata et quelques autres, dont Moïse Wadra, autour de la question: "Quelle sera la jeune musique kanak ?" Gilbert Téin, participant majeur, répond : "Elle sera jeune, c'est-à-dire maladroite, sans expérience, comme le comportement d'un jeune... Mais elle sera kanak! Influencé par ce mot 'kanak' au séminaire de Canala, tout le monde cherchait un nom à cette musique. Dans notre univers multilingue, on ne raisonne pas global mais plutôt chacun dans son terroir..."

Ainsi, Théo Menango propose une déclinaison moderne où K'n'K pourrait faire penser à Rock'n'Roll... Pourtant, Gilbert Téin reconnaît volontiers que c'est Moïse Wadra qui donna les reliefs de la sonorité du terme "kaneka". Moïse Wadra : "Si l'on enlève les deux voyelles de 'Kanak', on obtient $K n k$. Les politiques trouvaient cela confus. Mais j'ai écrit 'K est né K' et j'ai philosophé autour: quand le Kanak est né, il est devenu le premier-né, l'aîné de toutes les ethnies venues sur le territoire. Et quand un enfant sort du ventre de sa maman, pour dire qu'il vit, il faut qu'il pleure. Il émet un son et, s'il est l'aîné, c'est la première musique du pays. Voilà comment je définis le kaneka : K aîné K." 


\section{NOTES}

1. Melanesia 2000, première grande manifestation consacrée à la culture kanak dans la ville de Nouméa, du 3 au 7 septembre 1975, est décrite ainsi par Alban Bensa: "Sur une vaste esplanade surplombant la 'ville blanche', les Kanak de toutes les régions de l'archipel présentèrent avec un grand souci d'esthétique des exemples d'architecture mélanésienne, des objets artisanaux, des danses, etc., et des temps forts de la vie traditionnelle d'aujourd'hui : échanges cérémoniels entre clans, discours clamés par des spécialistes de l'art oratoire, chants de bienvenue, etc., autant d'expressions d'un art subtil de la sociabilité. En contrepoint, une évocation théâtrale de l'histoire du peuple kanak, incarné par le personnage de Kanaké, soulevait avec pudeur mais fermeté les problèmes sociaux et politiques posés par la colonisation : inégalités criantes, marginalisation des autochtones, etc."

"Chroniques kanak, l'ethnologie en marche", in Ethnies-Documents, vol. 10, n 18-19 (automne 1995), extrait de la communication "Vers Kanaky: tradition orale et idéologie nationaliste en Nouvelle-Calédonie”, prononcée en mars 1985.

\section{RÉSUMÉS}

Le kaneka, genre musical né en Nouvelle-Calédonie, s'est diffusé dans le Pacifique à partir des années 1990 (voir H\&M, n 1277). Sa création a fait partie de la stratégie de Jean-Marie Tjibaou pour la reconnaissance de l'identité culturelle des Kanak. Alors qu'en 2014 s'ouvrira la période de consultation électorale d'autodétermination prévue par les Accords de Nouméa, revenons sur ce qui a présidé à la naissance de cette nouvelle musique. 